
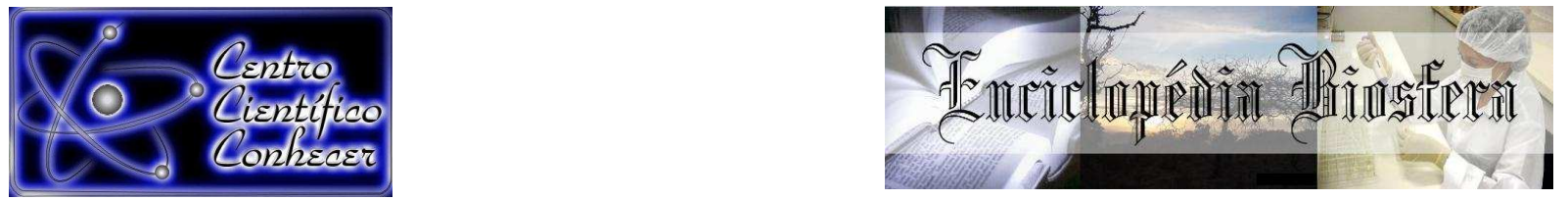

\title{
A EXPOSIÇÃO AO MERCÚRIO E OS EFEITOS DA EXPOSIÇÃO EM SERES HUMANOS NA PAN-AMAZÔNIA
}

\author{
Eloise de Sousa Cordeiro ${ }^{1}$, Altem Nascimento Pontes ${ }^{2}$, Veracilda Ribeiro Alves ${ }^{3}$, Cléa \\ Nazaré Carneiro Bichara ${ }^{4}$ \\ ${ }^{1}$ Licenciada em Química. Aluna especial do Programa de Pós-Graduação em Ciências \\ Ambientais da Universidade do Estado do Pará (UEPA), Belém, PA. Brasil. email: \\ eloise.ufpa@hotmail.com \\ ${ }^{2}$ Doutor em Ciências, na modalidade Física. Professor e Pesquisador do Programa de \\ Pós-Graduação em Ciências Ambientais da Universidade do Estado do Pará (UEPA), \\ Belém, PA. Brasil. \\ ${ }^{3}$ Doutora em Ciências Biológicas (Entomologia). Bolsista de Pós-doutorado do \\ Programa de Pós-Graduação em Ciências Ambientais da Universidade do Estado do \\ Pará (UEPA), Belém, PA. Brasil. \\ ${ }^{4}$ Graduada em Medicina. Doutorado em Biologia de Agentes Infecciosos e Parasitários. \\ Professora e Pesquisadora do Programa de Pós-Graduação em Ciências Ambientais da \\ Universidade do Estado do Pará (UEPA), Belém, PA. Brasil.
}

\section{Recebido em: 03/10/2016 - Aprovado em: 21/11/2016 - Publicado em: 05/12/2016 DOI: 10.18677/EnciBio 2016B 125}

\begin{abstract}
RESUMO
O mercúrio tem sido foco de preocupação global por causa da facilidade de ser transportado através da atmosfera, pela contaminação do meio ambiente e pela capacidade de bioacumulação nos ecossistemas, tendo impactos negativos sobre a saúde do meio ambiente e principalmente a saúde humana. Por este motivo, essa pesquisa teve como objetivo revisar artigos sobre o mercúrio na Pan-Amazônia, verificando os níveis de exposição e os efeitos da contaminação do mercúrio nas comunidades. O cabelo foi o biomarcador mais utilizado para as análises e as comunidades indígenas foram as mais estudadas. Foram investigados 40 artigos, sendo 30 divididos entre os países da Pan-Amazônia e 10 de Portarias de meio ambiente e vigilância ambiental. Foi feito um estudo de tendências de distribuição espacial, o qual confirmou que os níveis mais altos de exposição ao $\mathrm{Hg}^{0}$ são encontrados principalmente nas regiões indígenas e áreas de garimpos onde têm populações que consomem muito peixe. A análise de dados baseou-se em estudos de exposição e os efeitos comparados com os níveis de $\mathrm{Hg}^{\circ}$ conhecidos em diferentes países da Pan-Amazônia. O Brasil foi o país com maior número de artigos e Colômbia com a menor quantidade de trabalhos publicados nesse período. Os países da PanAmazônia têm dificuldade em detectar quais os países mais atingidos com contaminação por falta de divulgação das pesquisas, e a carência dessas informações dificulta a intervenção da vigilância em saúde para atuar junto às comunidades.
\end{abstract}

PALAVRAS-CHAVE: Contaminação. População. Peixe. 


\title{
EXPOSURE TO MERCURY AND THE EFFECTS OF EXPOSURE IN HUMANS IN THE PAN AMAZON
}

\begin{abstract}
The mercury has been the focus of global concern because of the ease of being transported through the atmosphere, the environmental contamination and the bioaccumulation capacity in ecosystems, having negative impacts on the health of the environment and especially to human health. For this reason, this research aimed to review articles on the mercury in the Pan-Amazon, checking the exposure levels and the effects of mercury contamination in communities. The hair was the most widely used biomarker for analysis and indigenous communities have been the most studied. The were investigated 40 articles, 30 divided between the Pan-Amazonian countries and 10 environmental ordinances and environmental monitoring. Was made study of trends spatial distribution, which confirmed that the highest levels of exposure to $\mathrm{Hg}^{\circ}$ are found mostly in indigenous regions and areas where mines have populations that consume a lot of fish. The Analysis data was based on studies exposure and effects compared to $\mathrm{Hg}^{\circ}$ levels known in different countries of the Pan-Amazon. The Brazil was the country with the largest number of articles and Colombia with the least amount of papers published in this period. Pan-Amazonian countries have difficulty detecting which countries most affected by contamination by lack of dissemination of research, and the lack of this information makes it difficult to health surveillance intervention to work with the communities.
\end{abstract}

KEYWORDS: Contamination. Population. Fish.

\section{INTRODUÇÃO}

O mercúrio é um metal pesado, prata, ubíquo e líquido à temperatura ambiente (MARRERO et al., 2011). Na sua forma pura, é conhecido como mercúrio elementar $(\mathrm{Hg} \circ$ ). Ele se volatiza facilmente formando vapores incolores e inodoros (GAIOLI et al., 2012). É um dos metais mais tóxicos existentes, podendo ser encontrado no ambiente tanto como consequência antropogênica resultante da atividade de mineração para extração de ouro, queima de combustíveis fósseis, indústrias siderúrgicas, incineração de produtos químicos, como advindos de fontes naturais do meio ambiente (VISNJEVEC et al., 2014).

Espécies de mercúrio existem também no ar ambiente, tanto na forma de vapor como nas fases de partículas associadas com os aerossóis e as nuvens, sendo que a principal espécie de mercúrio atmosférico é o mercúrio elementar $\left(\mathrm{Hg}^{\circ}\right)(\mathrm{TELMER}$ et al., 2009). Especula-se que há cerca de 6000 toneladas de mercúrio na atmosfera (ARIYA et al., 2015). Atualmente os níveis de mercúrio na atmosfera são entre três a seis vezes superiores aos níveis anteriores à industrialização (CHACON, 2002).

O mercúrio pode chegar até o ser humano através de exposição ocupacional e/ou ambiental (BUENO et al., 2011). O mercúrio metálico $\left(\mathrm{Hg}^{\circ}\right)$ pode formar vapores inodoros e incolores, com facilidade de penetração por vias respiratórias (GRIGOLETTO et al., 2008). Os garimpeiros artesanais podem ser considerados o grupo populacional que está diretamente exposto ao $\mathrm{Hg}$ ', devido o manuseio manual ou pela inalação dos vapores do mercúrio gerados no processo de queima do amálgama de mercúrio-ouro que pode ocorrer no interior de suas residências, gerando risco 
também para os familiares próximos (SILVA, 2011). É importante destacar a situação das mulheres que muitas vezes trabalham em tempo parcial na mineração informal, ocupam funções de cozinheiras e prestadoras de serviços, para muitas mulheres, a mineração artesanal significa uma oportunidade para tentar reduzir a pobreza (HINTON et al., 2003).

A exposição ambiental ocorre pela dieta alimentar, ao respirar ar contaminado, por ingestão de água e durante tratamentos dentários (CLARKSON et al., 2003; TRASANDE et al., 2010). As populações que vivem em regiões com contaminação por mercúrio especialmente as ribeirinhas e indígenas têm o pescado como fonte principal de proteínas e sofrem a exposição ambiental pela dieta alimentar (LEMIRE et al., 2006).

Independente da fonte, o mercúrio ao entrar em contato com ecossistemas aquáticos pode ser convertido em metilmercúrio $(\mathrm{MeHg})$ por bactérias anaeróbicas, sob certas condições ambientais (TINÔCO et al., 2010). O MeHg se infiltra nos organismos aquáticos e acumula-se alcançando a maior concentração possível no topo da cadeia trófica (ROJAS et al., 2010). Os peixes agem como bioacumuladores e dessa forma concentram e transferem resíduos da água para os humanos (DOREA, 2008).

É bom destacar que as gestantes, as lactantes e as crianças apresentam determinadas particularidades que aliadas às características do mercúrio tornam-se vulneráveis e são consideradas grupos de risco (DUTRA et al., 2012). Os compostos orgânicos de mercúrio podem ter efeitos adversos nesses grupos populacionais, uma vez que o metilmercúrio da mãe é transportado rapidamente pela barreira placentária até o feto, causando sérios danos, principalmente a nível neurológico (GUZZI \& PIGATTO, 2007).

Os alvos primários de toxicidade de mercúrio e seus compostos são o sistema nervoso, rins, sistema cardiovascular; os sistemas respiratórios, gastrointestinal, hematológico, imunológico, reprodutivo; sistemas de órgãos em desenvolvimento, como o sistema nervoso do feto, que são mais sensíveis aos efeitos tóxicos do mercúrio (UNEP, 2010). O mercúrio é uma preocupação global (DRISCOLL et al., 2013). O Programa das Nações Unidas para o Ambiente (UNEP) avaliou a carga de mercúrio global e estima que a mineração artesanal de pequena escala tem lançado aproximadamente $727 \mathrm{t}$ (toneladas métricas) de $\mathrm{Hg}$ para o ambiente por ano (UNEP, 2013). Isso é $37 \%$ do global de $1960 \mathrm{t}$ de $\mathrm{Hg}$ libertado anualmente por fontes antropogênicas para o meio ambiente (UNEP, 2013). Tais números são corroborados por vários estudos especificamente sobre mineração artesanal e de pequena escala de ouro (CORDY et al., 2011).

A Pan-Amazônia é composta por nove países que são Colômbia, Peru, Venezuela, Equador, Bolívia, Guiana, Guiana Francesa, Suriname e o Brasil, e todos têm em seu território a floresta amazônica, que é a maior floresta tropical e a bacia hidrográfica (PENNA FILHO, 2013;). O fato de os países da Pan-Amazônia serem ricos em biodiversidade e ter um vasto campo de recursos naturais faz com que se faça necessária a ampliação de estudos nesses países para que dessa forma a floresta amazônica seja preservada (RAVENA \& CAÑETE, 2007).

Devido suas características coletivas, o mercúrio é uma questão prioritária para diversos países da Pan-Amazônia, incluindo o Brasil, que assinaram um acordo gerado pela Convenção de Minamata em direção a um ambiente livre de mercúrio antropogênico (BRASIL, 2013). Apesar dos esforços, esse tipo de poluição continua a 
ser uma ameaça significativa nos países (BOSE-O'REILLY et al., 2010). Na PanAmazônia essa preocupação também tem crescido expressivamente e, por isso, a maior parte dos países desta região assinaram a Convenção de Minamata, a qual representa um importante avanço da comunidade internacional na implementação de medidas voltadas a proteger a saúde e o ambiente das emissões e liberações de mercúrio, dentre os países da Pan-Amazônia o único que não assinou a Convenção de Minamata foi o Suriname, porém dentre os países que assinaram somente a Bolívia, Guiana, Peru, e recentemente em julho de 2016 o Equador ratificaram (ROCHA NETO, 2013).

E isso é um problema para a sociedade, pois a ratificação é indispensável para que os países cumpram suas obrigações de direitos humanos, por que sem ela o meio ambiente e a população continuam sofrendo a exposição e contaminação por mercúrio (UNEP, 2014). Em países pertencentes à Pan-Amazônia, o processo de extração de ouro através da formação de amálgamas com o mercúrio é comumente utilizado, e isso é preocupante, e faz que se tenha uma atenção maior na questão de contaminação por mercúrio para estes países (CREMERS et al., 2013). Portanto, o objetivo deste trabalho foi fazer a revisão dos estudos atuais de exposição $\mathrm{Hg}^{\circ}$ na Pan-Amazônia, verificando quais as potenciais vias de exposição de $\mathrm{Hg}$, os biomarcadores e os efeitos do mercúrio para os moradores da Pan-Amazônia.

\section{MATERIAL E MÉTODOS}

Esse estudo baseou-se em pesquisa bibliográfica, exploratória e qualiquantitativa (GERHARDT \& SILVEIRA, 2009). Para realização deste trabalho foram utilizadas as bases de dados científicos e bibliotecas online, como Portal de Periódicos da Capes, ScienceDirect, SpringerLink, PubMed, SciELO e Biblioteca Virtual da Saúde.

Também os sites de instituições públicas internacionais, como a Agência de Proteção Ambiental (EPA), Sistemas de Vigilância Sanitária e de Vigilância em Saúde na América do Sul e a Organização Mundial da Saúde (OMS). Para a seleção de artigos, as palavras-chave foram: "Exposure Mercury, South American, Hair, Blood, Exposure Human, Fish".

A pesquisa foi realizada no período de julho de 2014 a junho de 2015. Foram excluídos os estudos que não definiram concentrações de $\mathrm{Hg}$ no cabelo, sangue, ou urina; não relataram informações críticas, como as áreas de estudo, número de amostras e tipos de população. Os artigos utilizados fazem estudos com ribeirinhos, homens, mulheres, crianças, indígenas e trabalhadores expostos com diferentes biomarcadores de exposição ( $\mathrm{Hg}$ no sangue, urina, ar e cabelo). A análise de dados foi baseada em estudos de exposição e os efeitos foram comparados com os níveis de $\mathrm{Hg}^{0}$ conhecidos em diferentes Países da Pan-Amazônia.

\section{RESULTADOS E DISCUSSÃO}

Foram investigados 40 artigos divididos entre os países: Bolívia: 4; Brasil: 9; Colômbia: 1; Equador: 3; Guiana Francesa: 2; Guiana: 0; Peru: 5; Suriname: 2; Venezuela: 4, 10 artigos de Portarias. A Figura 1 apresenta os resultados do levantamento bibliográfico de artigos sobre contaminação por mercúrio em cada país da Pan-Amazônia, nota-se que na Guiana não foram encontrados artigos sobre o assunto no período em que o levantamento foi feito. 


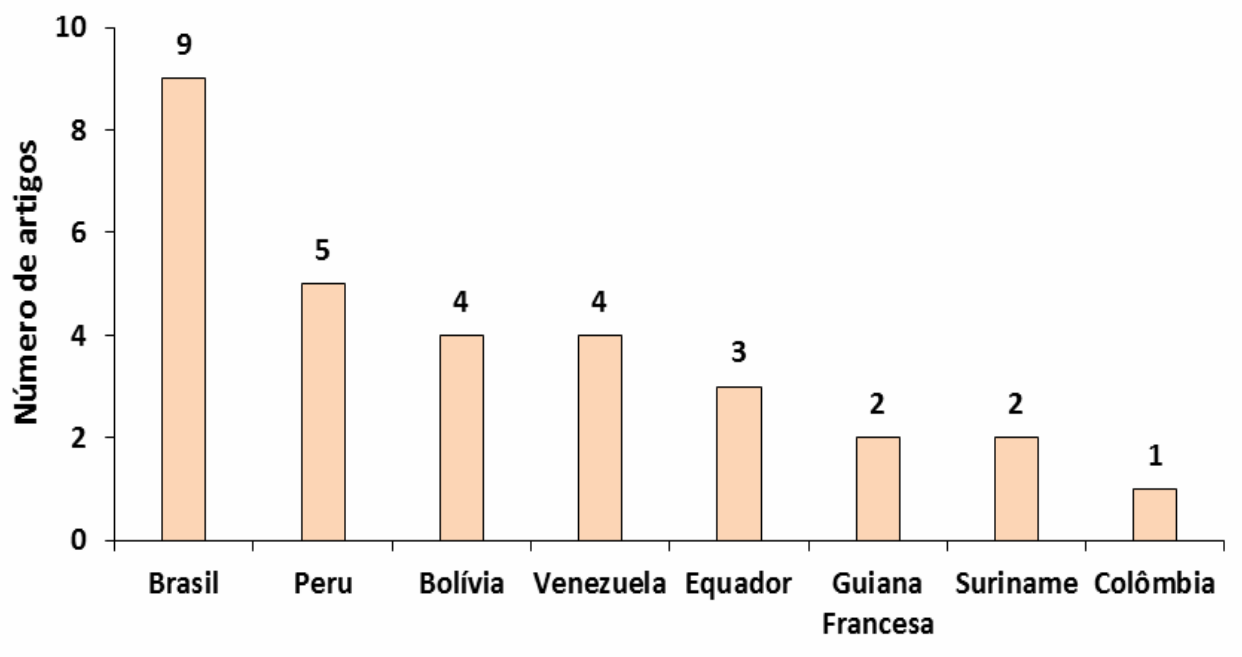

Países da Pan-Amazônia

FIGURA 1 - Levantamento bibliográfico de artigos no período entre julho de 2014 a junho de 2015 sobre contaminação em seres humanos por mercúrio na Pan-Amazônia. Fonte: Autores, 2016.

$\mathrm{Na}$ Figura 2, foram mostradas as bases de dados utilizadas para fazer 0 levantamento bibliográfico dos artigos sobre contaminação por mercúrio em seres humanos, em países da Pan-Amazônia. Nota-se que o PubMed sendo um recurso livre que é desenvolvido e mantido pelo National Center for Center for Biotechnology Information (NCBI), é o que possui maior quantidade de artigos de acesso livre com relação ao mercúrio. Os demais, como pode ser observado na Figura 2, possuem menos artigos livres, sendo que na base SpringerLink a maior parte dos artigos encontrados são de acesso restrito, fato que dificultou a pesquisa teórica.

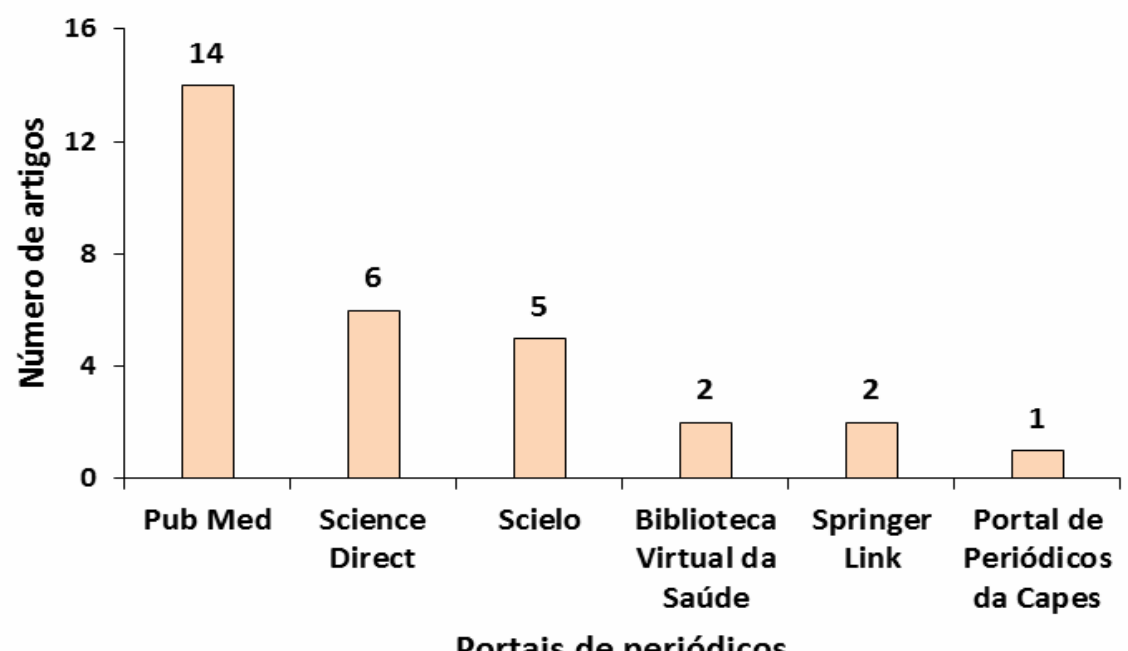

FIGURA 2 - Portais de periódicos utilizados no levantamento bibliográfico de artigos sobre contaminação por mercúrio no período de entre julho de 2014 a junho de 2015. Fonte: Autores, 2016. 
As bases de dados como Sistema de Informação de Vigilância em Saúde de Populações Expostas a Solo Contaminado (SISSOLO) e Sistema de Informação de Agravos de Notificação (SINAN) fazem o levantamento sobre o cadastramento de contaminação por mercúrio que são encontradas no Brasil e tem o intuito de proteger a saúde das pessoas afetadas e informar as pessoas expostas ao mercúrio.

Existem decretos para o controle da produção e comercialização de substâncias que contenham o mercúrio, isso é feito para proteger o meio ambiente e a saúde humana. Porém, muitos países ainda têm altos níveis de contaminação como é o caso do Brasil e Colômbia, no Brasil isso pode ser verificado através dos sistemas nacionais de informação existentes como o Sistema Nacional de Agravos de Notificações (SINAN) seguido pelo Sistema de Informação sobre Nascidos Vivos (SISNAC), o Sistema de Informação de Mortalidade (SIM) e pelo Centro de Informações Estratégias em Vigilância em Saúde (CIEVS), na Colômbia a notificação efetiva é feita através de protocolos de monitoramentos e controle de intoxicação aguda que é desenvolvido no Instituto Nacional de Saúde, que faz o acompanhamento continuo dos casos encontrados no país (LEMOS et al., 2011).

A Venezuela, apesar de obter muitas legislações de proteção ao meio ambiente, ainda se tem verificado vários problemas em relação ao meio ambiente que até hoje não foram resolvidos (MARRERO et al., 2011). No quadro 1, pode se verificar essas questões. Existe a ausência de atitudes translacionais como o SISSOLO e SINAN que acarreta a falta de unidade entre os países da Pan-Amazônia. O SISSOLO é um sistema de informação que serve de instrumento para subsidiar as ações da Vigilância em Saúde, no qual é feito um cadastro onde estão localizadas as áreas com populações expostas ou potencialmente expostas a contaminantes químicos, e o SINAN que é um sistema de informação alimentado pela notificação e investigação de casos de doenças e agravos que constam da lista nacional de doenças de notificação compulsória (Portaria GM/MS № 2325 de 8 de dezembro de 2003). 
QUADRO 1 - Descrição dos artigos sobre exposição dos seres humanos ao mercúrio na Pan-Amazônia

\begin{tabular}{|c|c|c|c|c|c|}
\hline & +2 & Biomarcador & $\begin{array}{l}\text { Pessoas } \\
\text { analisadas }\end{array}$ & Fonte de contaminação & População \\
\hline \multirow[t]{4}{*}{ Bolívia } & $\begin{array}{l}\text { A nutritional dilemma: fish } \\
\text { consumption, } \\
\text { exposure and growth of } \\
\text { children in Amazonian Bolivia } \\
\text { (BENEFICE et al., 2008). }\end{array}$ & Cabelo & $\begin{array}{l}\text { Mulheres e } \\
\text { Crianças }\end{array}$ & $\begin{array}{l}\text { O peixe, a população tem como } \\
\text { principal fonte de proteína. }\end{array}$ & Urbana \\
\hline & $\begin{array}{l}\text { Fishing activity, health } \\
\text { characteristics and mercury } \\
\text { exposure of Amerindian } \\
\text { women living alongside the } \\
\text { Beni River (Amazonian } \\
\text { Bolivia) (BENEFICE et al., } \\
\text { 2010). }\end{array}$ & Cabelo & Mulheres & $\begin{array}{l}\text { O peixe, a população tem como } \\
\text { principal fonte de proteína. }\end{array}$ & Indígena \\
\hline & $\begin{array}{l}\text { Mercury exposure in a high } \\
\text { fish eating Bolivian } \\
\text { Amazonian population with } \\
\text { intense small-scale gold- } \\
\text { mining activities (BARBIERE } \\
\text { et al., 2009). }\end{array}$ & Cabelo & Ambos gênero & $\begin{array}{l}\text { O peixe, a população tem como } \\
\text { principal fonte de proteína. }\end{array}$ & Indígena \\
\hline & $\begin{array}{l}\text { Lifestyle and } \text { mercury } \\
\text { contamination of Amerindian } \\
\text { populations along the Beni } \\
\text { river (lowland Bolivia) } \\
\text { (MONRROY et al., 2008). }\end{array}$ & Cabelo & $\begin{array}{l}\text { Mulheres e } \\
\text { filhos adultos }\end{array}$ & $\begin{array}{l}\text { O peixe, a população tem como } \\
\text { principal fonte de proteína. }\end{array}$ & Indígena \\
\hline \multirow[t]{4}{*}{ Brasil } & $\begin{array}{l}\text { Fish consumption and } \\
\text { bioindicators of inorganic } \\
\text { mercury exposure (PASSOS } \\
\text { et al., 2007). }\end{array}$ & Sangue e urina & Ambos gênero & $\begin{array}{l}\text { O peixe, a população tem como } \\
\text { principal fonte de proteína. }\end{array}$ & Indígena \\
\hline & $\begin{array}{l}\text { Total hair mercury in children } \\
\text { from a coastal population in } \\
\text { Cananéia, São Paulo State, } \\
\text { Brazil (FARIAS et al., 2008). }\end{array}$ & Cabelo & $\begin{array}{l}\text { Crianças } \\
\text { (Ambos } \\
\text { gênero) }\end{array}$ & $\begin{array}{l}\text { O peixe, pois a população tem } \\
\text { como principal fonte de proteína. }\end{array}$ & Indígena \\
\hline & $\begin{array}{l}\text { Exposure to organic mercury } \\
\text { in riparian populations on the } \\
\text { Upper Madeira river, } \\
\text { Rondonia, Brazil, 1991: } \\
\text { preliminary } \\
\text { (BOISCHIO e BARBOSA, } \\
\text { 1993). }\end{array}$ & Cabelo & $\begin{array}{l}\text { Crianças e } \\
\text { adultos }\end{array}$ & $\begin{array}{l}\text { O peixe, a população tem como } \\
\text { principal fonte de proteína. }\end{array}$ & Indígena \\
\hline & $\begin{array}{l}\text { Hair mercury levels in } \\
\text { Amazonian populations: } \\
\text { spatial distribution and trends }\end{array}$ & Cabelo & Ambos gênero & $\begin{array}{l}\text { O peixe, a população tem como } \\
\text { principal fonte de proteína. }\end{array}$ & Indígena \\
\hline
\end{tabular}

ENCICLOPÉDIA BIOSFERA, Centro Científico Conhecer - Goiânia, v.13 n.24; p.

13532016




\begin{tabular}{|c|c|c|c|c|c|}
\hline & $\begin{array}{l}\text { (BARBIERE e GARDON, } \\
\text { 2009). }\end{array}$ & & & & \\
\hline & $\begin{array}{l}\text { Hearing thresholds in children } \\
\text { exposed to mercury in the } \\
\text { prenatal period (DUTRA et al., } \\
\text { 2012). }\end{array}$ & $\begin{array}{l}\text { Cordão } \\
\text { Umbilical }\end{array}$ & $\begin{array}{l}\text { Crianças } \\
\text { (Ambos } \\
\text { gênero) }\end{array}$ & $\begin{array}{l}\text { O peixe, a população tem como } \\
\text { principal fonte de proteína. }\end{array}$ & Urbana \\
\hline & $\begin{array}{l}\text { Mercury exposure among } \\
\text { Pakaanóva Indians, Amazon } \\
\text { Region, Brazil (SANTOS et } \\
\text { al., 2003). }\end{array}$ & Cabelo & $\begin{array}{l}\text { Crianças e } \\
\text { adultos }\end{array}$ & $\begin{array}{l}\text { O peixe, a população tem como } \\
\text { principal fonte de proteína. }\end{array}$ & Indígena \\
\hline & $\begin{array}{l}\text { Mercury levels in fish } \\
\text { consumed by the Sai Cinza } \\
\text { indigenous } \\
\text { Munduruku community, } \\
\text { Jacareacanga County, State } \\
\text { of Pará, Brazil (BRABO et al., } \\
\text { 1999). }\end{array}$ & $\begin{array}{l}\text { Músculo de } \\
\text { peixe }\end{array}$ & Ambos gênero & $\begin{array}{l}\text { O peixe, a população tem como } \\
\text { principal fonte de proteína. }\end{array}$ & Indígena \\
\hline & $\begin{array}{l}\text { Maternal mercury exposure } \\
\text { and neuro-motor development } \\
\text { in breastfed infants from Porto } \\
\text { Velho (Amazon), Brazil } \\
\text { (MARQUES et al., 2007). }\end{array}$ & $\begin{array}{l}\text { Cabelo, Cordão } \\
\text { Umbilical e } \\
\text { sangue }\end{array}$ & $\begin{array}{l}\text { Mulheres e } \\
\text { recém- } \\
\text { nascidos }\end{array}$ & $\begin{array}{l}\text { O peixe, a população tem como } \\
\text { principal fonte de proteína. }\end{array}$ & Urbana \\
\hline & $\begin{array}{l}\text { Methylmercury human } \\
\text { exposure in riverine villages of } \\
\text { Tapajos basin, Pará State, } \\
\text { Brazil (PINHEIRO et al., } \\
\text { 2000). }\end{array}$ & Cabelo & Ambos gênero & $\begin{array}{l}\text { O peixe, a população tem como } \\
\text { principal fonte de proteína. }\end{array}$ & Urbana \\
\hline Colômbia & $\begin{array}{l}\text { Mercury contamination from } \\
\text { artisanal gold mining in } \\
\text { Antioquia, Colombia: The } \\
\text { world's highest per capita } \\
\text { mercury pollution (CORDY et } \\
\text { al., 2011). }\end{array}$ & Cabelo & Ambos gênero & $\begin{array}{l}\text { Mineração, a população esta } \\
\text { exposta através da queima de } \\
\text { amálgama. }\end{array}$ & Urbana \\
\hline Equador & $\begin{array}{l}\text { Air Mercury Contamination in } \\
\text { the Gold Mining Town of } \\
\text { Portovelo, } \\
\text { (GONZÁLEZ-CARRASCO Ecuador } \\
\text { al., 2011). }\end{array}$ & $\mathrm{Ar}$ & - & $\begin{array}{l}\text { Mineração artesanal de ouro, a } \\
\text { população esta exposta através } \\
\text { da queima de amálgama. }\end{array}$ & Urbana \\
\hline & $\begin{array}{l}\text { Exposure and toxic effects of } \\
\text { elemental mercury in gold- } \\
\text { mining activities in Ecuador } \\
\text { (HARARI et al., 2012). }\end{array}$ & Sangue e Urina & Homens & $\begin{array}{l}\text { Mineração artesanal de ouro, a } \\
\text { população esta exposta através da } \\
\text { queima de amálgama. }\end{array}$ & Indígena \\
\hline & $\begin{array}{l}\text { Neurocognitive Screening of } \\
\text { Mercury-exposed Children of }\end{array}$ & $\begin{array}{l}\text { Cabelo e } \\
\text { Sangue }\end{array}$ & Crianças & $\begin{array}{l}\text { Mineração, a população esta } \\
\text { exposta através da queima de }\end{array}$ & Indígena \\
\hline
\end{tabular}

ENCICLOPÉDIA BIOSFERA, Centro Científico Conhecer - Goiânia, v.13 n.24; p. 


\begin{tabular}{|c|c|c|c|c|c|}
\hline & $\begin{array}{l}\text { Andean Gold Miners } \\
\text { (COUNTER et al., 2006). }\end{array}$ & & & amálgama. & \\
\hline \multirow[t]{2}{*}{ Guiana Francesa } & $\begin{array}{l}\text { Gold-Mining Activities and } \\
\text { Mercury Contamination of } \\
\text { Native Amerindian } \\
\text { Communities in French } \\
\text { Guiana: Key Role of Fish in } \\
\text { Dietary Uptake (FRÉRY et } \\
\text { al.,2001). }\end{array}$ & $\begin{array}{l}\text { Cabelo e } \\
\text { Músculo de } \\
\text { peixe }\end{array}$ & Ambos gênero & $\begin{array}{l}\text { Peixe, a população tem como } \\
\text { principal fonte de proteína. }\end{array}$ & Indígena \\
\hline & $\begin{array}{l}\text { Mercury Contamination in } \\
\text { Humans in Upper Maroni, } \\
\text { French Guiana Between } 2004 \\
\text { and } 2009 \text { (FUJIMURA et al., } \\
2012 \text { ). }\end{array}$ & $\begin{array}{l}\text { Cabelo e } \\
\text { Músculo de } \\
\text { peixe }\end{array}$ & Ambos gênero & $\begin{array}{l}\text { Peixe, a população tem como } \\
\text { principal fonte de proteína. }\end{array}$ & Indígena \\
\hline \multirow[t]{5}{*}{ Peru } & 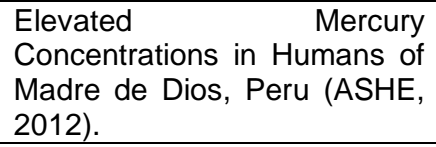 & Cabelo & Ambos gênero & $\begin{array}{l}\text { Mineração, a população esta } \\
\text { exposta através da queima de } \\
\text { amálgama. }\end{array}$ & Indígena \\
\hline & $\begin{array}{l}\text { Enfermedades transmisibles, } \\
\text { salud mental y exposición a } \\
\text { contaminantes ambientales } \\
\text { en población aledaña al } \\
\text { proyecto minero las bambas } \\
\text { antes de la fase de } \\
\text { explotación, Perú } 2006 \\
\text { (STETE et al., 2010). }\end{array}$ & Urina & $\begin{array}{l}\text { Crianças e } \\
\text { adultos }\end{array}$ & $\begin{array}{l}\text { Mineração, a população esta } \\
\text { exposta através da queima de } \\
\text { amálgama. }\end{array}$ & Indígena \\
\hline & $\begin{array}{l}\text { Mercury Exposure Among } \\
\text { Artisanal Gold Miners in } \\
\text { Madre de Dios, Peru: A } \\
\text { Cross-sectional Study (YARD } \\
\text { et al., 2012). }\end{array}$ & Urina e Sangue & Ambos gênero & $\begin{array}{l}\text { Mineração, a população esta } \\
\text { exposta através da queima de } \\
\text { amálgama. }\end{array}$ & Urbana \\
\hline & $\begin{array}{l}\text { Mercury Exposure in Informal } \\
\text { Gold Miners and Relatives in } \\
\text { Southern Peru (HURTADO et } \\
\text { al., 2013). }\end{array}$ & Urina e Ar & Ambos gênero & $\begin{array}{l}\text { Mineração informal, fusão e } \\
\text { fundição de amálgama. }\end{array}$ & Urbana \\
\hline & $\begin{array}{l}\text { Residential Mercury } \\
\text { Contamination in Adobe Brick } \\
\text { Homes in Huancavelica, Peru } \\
\text { (HAGAN et al., 2013). }\end{array}$ & - & - & $\begin{array}{l}\text { Mineração artesanal de ouro, a } \\
\text { população esta exposta através } \\
\text { da queima, fusão e fundição da } \\
\text { amálgama. }\end{array}$ & Urbana \\
\hline Suriname & 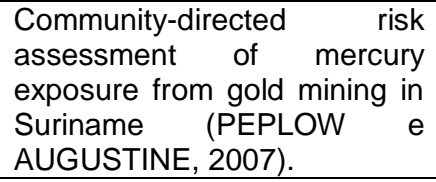 & Cabelo & Ambos gênero & $\begin{array}{l}\text { Peixe, a população tem como } \\
\text { principal fonte de proteína. }\end{array}$ & Indígena \\
\hline
\end{tabular}

ENCICLOPÉDIA BIOSFERA, Centro Científico Conhecer - Goiânia, v.13 n.24; p. 


\begin{tabular}{|c|c|c|c|c|c|}
\hline & $\begin{array}{l}\text { Community-Led Assessment } \\
\text { of Risk from Exposure to } \\
\text { Mercury by Native Amerindian } \\
\text { Wayana in Southeast } \\
\text { Suriname (PEPLOW e } \\
\text { AUGUSTINE, 2012). }\end{array}$ & Cabelo & Ambos gênero & $\begin{array}{l}\text { Peixe, a população tem como } \\
\text { principal fonte de proteína. }\end{array}$ & Indígena \\
\hline \multirow[t]{5}{*}{ Venezuela } & $\begin{array}{llr}\text { Biological } & \text { monitoring } & \text { of } \\
\text { mercury } & \text { exposure } & \text { in } \\
\text { individuals referred to a } \\
\text { toxicological center } \\
\text { Venezuela (ROJAS et al., } \\
\text { 2006). }\end{array}$ & Urina & $\begin{array}{l}\text { Crianças e } \\
\text { adultos }\end{array}$ & $\begin{array}{l}\text { Mineração artesanal de ouro, a } \\
\text { população esta exposta através da } \\
\text { queima de amálgama. }\end{array}$ & Indígena \\
\hline & $\begin{array}{l}\text { Exposición ambiental al } \\
\text { mercurio y valores en orina de } \\
\text { los habitantes de la } \\
\text { comunidad Boca de Yaracuy, } \\
\text { ubicada en la costa centro- } \\
\text { norte de Venezuela } \\
\text { (MARRERO et al., 2011). }\end{array}$ & Urina & Ambos gênero & $\begin{array}{l}\text { Alto nível de } \mathrm{Hg}^{\circ} \text { na urina, mas } \\
\text { ainda não se sabe qual o fator } \\
\text { ambiental relacionado com este } \\
\text { metal. }\end{array}$ & Indígena \\
\hline & $\begin{array}{lr}\text { Exposición } & \text { ambiental } \\
\text { humana al } & \text { mercurio en } \\
\text { Venezuela: } & 2004-2008 \\
\text { (ROJAS, 2010). } & \\
\end{array}$ & $\begin{array}{l}\text { Cabelo, Urina e } \\
\text { Sangue }\end{array}$ & Ambos gênero & $\begin{array}{l}\text { O consumo de peixe e mineração } \\
\text { artesanal de ouro. }\end{array}$ & Urbana \\
\hline & $\begin{array}{l}\text { Occupational exposure to } \\
\text { airborne mercury during gold } \\
\text { mining operations near El } \\
\text { Callao, Venezuela (DRAKE et } \\
\text { al., 2011). }\end{array}$ & Urina & Ambos gênero & $\begin{array}{l}\text { Mineração, queima da amálgama } \\
\text { de mercúrio de ouro, refino de ouro } \\
\text { e fundição. }\end{array}$ & Urbana \\
\hline & $\begin{array}{l}\text { Presencia de mercurio total } \\
\text { en habitantes de los } \\
\text { asentamientos Indígenas el } \\
\text { casabe, municipio autónomo } \\
\text { raúl leoni y el plomo, } \\
\text { Municipio autónomo MANUEL } \\
\text { CARLOS PIAR - ESTADO } \\
\text { Bolívar (ÁLVAREZ e ROJAS, } \\
\text { 2006). }\end{array}$ & Cabelo, Urina & Ambos gênero & $\begin{array}{l}\text { O consumo de peixe e mineração } \\
\text { artesanal de ouro. }\end{array}$ & Indígena \\
\hline
\end{tabular}

Fonte: Autores, (2016). 
Apesar da mineração de ouro ser artesanal e de pequena escala ser a principal fonte de poluição por $\mathrm{Hg}$, essa atividade também é a mais importante para a população de baixa renda (GONZÁLEZ-CARRASCO et al., 2011) e na maioria das vezes é a única fonte de renda para muitas pessoas que vivem nos países da Pan-Amazônia que fazem mineração artesanal de pequena escala.

A Bolívia faz um levantamento em relação à população urbana e principalmente indígena, os estudos levaram em consideração todos os gêneros e a idade, o cabelo foi biomarcador utilizado para as análises de indicadores de contaminação relacionadas ao consumo de peixe e exposição a áreas de mineração (PASSOS \& MERGLER, 2008).

Observou-se em todos os artigos pesquisados que essas comunidades indígenas se alimentam quase que exclusivamente por peixe. Atualmente sabe-se que o consumo de peixe e uma via de contaminação por $\mathrm{Hg}^{\circ}$. A questão que gera preocupação é a importância do consumo de peixe na alimentação por causa dos nutrientes que ele contém (BARBIERE et al., 2009).

Entre as mulheres pesquisadas as mais afetadas pelo $\mathrm{Hg}^{\circ}$ foram as que vivem as margens do rio. Muitas relataram um alto índice de abortos espontâneos e questões relacionadas à saúde dos bebês que conseguiram sobreviver, além de bebês que nasceram e morrem logo após o parto ou até mesmo tiveram fetos natimortos (MONRROY et al., 2008).

Na Colômbia o estudo encontrado mostra o problema em relação a migração da mineração para os centros urbanos, estes centros atualmente são os locais onde ocorrem a amalgamação de todo o minério, onde o minério é queimado sem qualquer sistema de filtração. Porém, o que torna uma questão preocupante é o modo que é feito a extração, sem nenhuma assistência técnica ocorre de forma rudimentar e isso gera impactos ambientais e de saúde para as pessoas que fazem a manipulação e também para as que convivem por perto de onde ocorre a extração (CORDY et al., 2011).

O Suriname tem a pesquisa bem semelhante a da Bolívia e Colômbia, pois ambos utilizaram o mesmo biomarcador, a população de estudo foi de ambos os gêneros, indígenas e tem um alto índice de consumo de peixe. $E$ a dificuldade encontrada na coleta foi à questão cultural desses povos, por isso achou-se melhor e mais prático fazer análise do cabelo (PEPLOW \& AUGUSTINE, 2007).

Deste país analisaram-se dois artigos que tecem críticas sobre a questão de saúde pública nos países da Pan-Amazônia, uma problemática que há muito tempo se tenta solucionar, é a questão da contaminação por mercúrio em comunidades expostas. Nesta pesquisa os autores fizeram estudos com comunidades indígenas, e desenvolveu o processo da pesquisa para que as próprias comunidades com ajuda de líderes comunitários pudessem verificar uma possível contaminação por $\mathrm{Hg}^{\circ}$ (BOVEN, 2006; PEPLOW \& AUGUSTINE, 2012).

O Suriname tem poucos artigos publicados em relação à avaliação de risco e os efeitos da poluição do $\mathrm{Hg}$, fato este que dificulta o mapeamento com a localização de exposição dessas comunidades. Na Venezuela a urina foi o biomarcador utilizado em todos os artigos, mas também se utilizou o cabelo e sangue, nota-se que as pesquisas se concentraram em adultos de ambos os gêneros e que pouco se aborda em relação às crianças versus contaminação por mercúrio (ÁLVAREZ \& ROJAS, 2006; ROJAS et al., 2006; ROJAS, 2010; DRAKE et al., 2011; MARRERO et al., 2011). 
Esse país tem um Centro de Investigações Toxicológicas da Universidade de Carabobo (CITUC) que é o local onde são feitas a maioria das pesquisas toxicológicas. Eles têm uma base científica onde estão localizados os dados que contribuem para a prevenção e controle dos efeitos químicos. O apoio técnico aos programas profissionais ou da comunidade relacionada à exposição, são essas ações efetivas que este país tem e que faltam nos demais países para solucionar a questão de contaminação e exposição ao mercúrio (BARREQARD, 2010).

Apesar de muitos avanços ainda é necessário um estudo mais aporfundado para obter uma melhor caracterização dos riscos de exposição mercurial no país, pois os resultados mostraram dados importantes para futuros estudos sobre o impacto do $\mathrm{Hg}^{\circ}$.

No Equador os biomarcadores utilizados foram o ar, cabelo, sangue e urina. Artigos fizeram estudos com homens e chegaram à conclusão de que pessoas que comercializam ouro têm uma exposição muito maior de riscos do que os mineiros, nos quais a exposição varia ao longo do tempo. As crianças pesquisadas têm o risco de neurocognitivo adverso, por causa do nível de contaminação que também deve esta relacionada à dieta alimentar dessa população urbana (COUNTER et al., 2006).

Foram avaliadas concentrações do mercúrio no ar de Portovelo local onde tem uma das mais antigas minas de ouro e que ainda utiliza o processo de mineração artesanal para a recuperação de ouro. E encontrados altos níveis de mercúrio em El Pache, através desses estudos se verificou que na área urbana foram detectados níveis mais baixos, o que mostra que possivelmente na região rural do Equador é onde tem o maior índice de mineração irregular e rudimentar (GONZÁLEZ-CARRASCO et al., 2011).

A Guiana Francesa faz um levantamento em relação à população indígena, onde os estudos levaram em consideração ambos os gêneros, e o cabelo e músculo de peixe foram os biomarcadores utilizados para as análises de indicadores de contaminação. Os resultados mostram que o mercúrio alimentar assim como em todos os países da Pan-Amazônia é a principal fonte de contaminação (FUJIMURA et al, 2011).

O Peru é a parte da Amazônia que tem um foco crescente de mineração artesanal, por este motivo existe uma grande preocupação com a contaminação por mercúrio ambiental que vem ocorrendo principalmente em Madre Dios, local onde assim como no Equador ainda tem muitos mineiros artesanais (ASHE, 2012).

Neste país foi difícil obter um resultado concreto das análises, pois Madre Dios não tem laboratórios avançados para as análises e um diagnóstico efetivo. Essa comunidade foi uma das primeiras investigadas para medir a concentração de mercúrio de mineração de ouro artesanal, utilizando tanto urina e sangue como biomarcadores.

No Brasil sete artigos utilizados na revisão estudam comunidades indígenas e os biomarcadores usados foram cabelo, sangue, urina e tiveram as pesquisas voltadas para adultos e crianças de ambos os gêneros. O Brasil é o país da Pan-Amazônia que tem mais estudos voltados para a problemática da questão de exposição e contaminação por mercúrio, principalmente em regiões de garimpos e áreas indígenas, porém, poucos estudos têm-se centrado na descoberta de biomarcadores de mercúrio na população da região. Foram encontrados estudos realizados em pessoas de sete comunidades ribeirinhas do Rio Tapajós, sem história de exposição de mercúrio inorgânico da ocupação ou amálgamas dentárias (PINHEIRO et al., 2000). Aplicou-se um questionário para saber a rotina dessa população e depois de analisadas as 
amostras de sangue e urina por espectrometria de absorção atômica, os resultados indicaram evidências para a relação entre o consumo de peixe e a contaminação nas pessoas que se alimentam do mesmo, pois as comunidades ribeirinhas são maiores consumidoras de peixe (PASSOS \& MERGLER, 2008).

\section{CONCLUSÃO}

Esta pesquisa mostrou a ausência de um sistema de notificação que produza um elo unificando os países da Pan-Amazônia, através do compartilhamento de dados sobre os estudos de exposição humana ao mercúrio, para que todos tenham acesso à informação em relação aos níveis de exposição.

Verificou-se a necessidade da criação de programas de intercalibração entre as unidades de referência credenciadas, a padronização de metodologias analíticas de quantificação de mercúrio e a elaboração de uma lista de países com casos de contaminação especificando cidades e áreas afetadas, compartilhando as técnicas e metodologias analíticas para a obtenção do controle preciso e de qualidade.

Esses países necessitam de mais investimento em pesquisa e divulgação sobre a exposição e a contaminação ao mercúrio. A carência dessas informações dificulta a intervenção da vigilância em saúde na atuação junto às comunidades para o diagnóstico consolidado de contaminação.

\section{AGRADECIMENTOS}

Ao Programa de Pós-Graduação em Ciências Ambientais, em nível de Mestrado Acadêmico, ofertado pela Universidade do Estado do Pará (UEPA), na cidade de Belém-PA. Agradeço pela oportunidade, incentivo e o apoio nesta pesquisa.

\section{REFERÊNCIAS}

ÁLVAREZ, L.; ROJAS, L. Presencia de mercurio total en habitantes de los asentamientos Indígenas el casabe, municipio autónomo raúl leoni y el plomo, Municipio autónomo MANUEL CARLOS PIAR - ESTADO Bolívar. Saber, Universidad de Oriente, Venezuela, v.18. n.2: p. 161-167, 2006. Disponível em: < http://www.redalyc.org/articulo.oa?id=427739430008>.

ASHE, K. Elevated Mercury Concentrations in Humans of Madre de Dios, Peru. PLoS

ONE. v. 7, ISSUE 3, 2012. Disponível em: < https://www.ncbi.nlm.nih.gov/pmc/articles/PMC3306380/pdf/pone.0033305.pdf >.

BARBIERE, F. L.; COURNIL, A.; GARDON, J. Mercury exposure in a high fish eating Bolivian Amazonian population with intense small-scale gold-mining activities. International Journal of Environmental Health Research, v. 19, n. 4, p. 267-277, 2009. Disponível em:< http://dx.doi.org/10.1080/09603120802559342>.

BARBIERE, F. L.; GARDON, J. Hair mercury levels in Amazonian populations: spatial distribution and trends. International Journal of Health Geographics, v. 19, n. 4, p. 267-277, 2009. Disponível em: < DOI: 10.1186/1476-072X-8-71>.

BARREQARD, L. Biological monitoring of exposure to mercury vapor . by Barregard $L$ Affiliation $\square$ : Department of Occupational Medicine, Sahlgren's Biological monitoring of ENCICLOPÉDIA BIOSFERA, Centro Científico Conhecer - Goiânia, v.13 n.24; p. 1359 2016 
exposure to mercury vapor. Scandinavian Journal of Work, Environment \& Health, v. 36, n. 3, p. 45-49, 2010.

BENEFICE, E.; LUNA-MONRROY, S.; LOPEZ-RODRIGUEZ, R. Fishing activity, health characteristics and mercury exposure of Amerindian women living alongside the Beni River (Amazonian Bolivia). International Journal of Hygiene and Environmental Health, v. 213, n. 6, p. 458-464, 2010. Elsevier GmbH. Disponível em: <http://dx.doi.org/10.1016/j.ijheh.2010.08.010>.

BENEFICE, E.; MONRROY, S.J.; RODRIGUEZ, R.W. A nutritional dilemma: fish consumption, mercury exposure and growth of children in Amazonian Bolivia. International Journal of Environmental Health Research. v. 18, n. 6, p. 415-427, 2008. Disponível em: < http://dx.doi.org/10.1080/09603120802272235>.

BOSE-O'REILLY, S.; MCCARTY, K.; STECKLING, N.; LETTMEIER, B. Mercury exposure and children's health. Current Problems in Pediatric and Adolescent Health Care, v. 40, n. 8, p. 186-215, 2010.

BOISCHIO, A. A. P.; BARBOSA, A. Exposição ao mercúrio orgânico em populações ribeirinhas do Alto Madeira, Rondônia, 1991: resultados preliminares. Caderno de Saúde Pública, v. 9 , n. 2, p. 155-160, 1993. Disponível em:< http://dx.doi.org/10.1590/S0102-311X1993000200006>.

BOVEN, K. Overlaven no en Grengsebied: Veranderingsprocessen Bij de Wayana no Suriname en Frans-Guyana. Amsterdam, the Netherlands: IBS Publishers; 2006.

BRABO, E. S.; SANTOS, E. C.; JESUS, I. M.; MASCARENHAS, A.F.; FAIAL, K.F. Mercury levels in fish consumed by the Sai Cinza indigenous community, Munduruku Reservation, Jacareacanga County, State of Pará, Brazil. Caderno de Saúde Pública, v. 15, n. 2, p. 325-332, 1999. Disponível em:< http://dx.doi.org/10.1590/S0102$311 \times 1999000200017>$.

BUENO, P. C.; RODRIGUES, J. C.; LEMOS, A. F.; MALASPINA, F.G.; MATSUI, C.T.; ROHLFS, D.B. Exposição humana a mercúrio: subsídios para o fortalecimento das ações de vigilância em saúde. Caderno de Saúde Coletiva, Rio de Janeiro, v. 4, n. 19, p. 433-447, 2011. Disponível em: <http://www.iesc.ufrj.br/cadernos/images/csc/2011_4/artigos/csc_v19n4_443-447.pdf>.

CORDY, P.; VEIGA, M. M.; SALIH, I.; AL-SAADI, S.; CONSOLE, S.; GARCIA, O.; MESA, L.A.; VELÁSQUEZ-LÓPEZ, P.C.; ROESER, M. Mercury contamination from artisanal gold mining in Antioquia, Colombia: The world's highest per capita mercury pollution. Science of the Total Environment, v. 410-411, p. 154-160, 2011. Elsevier B.V. Disponível em: <http://dx.doi.org/10.1016/j.scitotenv.2011.09.006>.

COUNTER, S. A.; BUCHANAN, L. H.; ORTEGA, F. Neurocognitive Screening of Mercury-exposed Children of Andean Gold Miners, International Journal of 
Occupational and Environmental Health, v. 12, n. 3, p. 209-214, 2006. Disponível em: < DOl: 10.1179/ oeh.2006.12.3.209>.

CLARKSON, T.W.; MAGOS, L.; GARY J. MYERS, M. D. The toxicology of Mercury current exposures and clinical manifestations, The New England Journal of Medicine, v. 349, p. 1731-1737, 2003.

CHACON, E. A. Proyecto $\square$ : Tipo de Informe $\square$ : Número de Serie $\square$ : UTF / COL / 024 Informe Técnico INF_IB. Programa De Desarrollo Sostenible De La Region De La Mojana "Infraestructura Basica Organización de las Naciones Unidas para la Agricultura y la Alimentación, San ., p. 1-141, 2002.

CREMERS, L.; KOLEN, J.; THEIJE, M. CEDLA, Centre for Latin American Studies and Documentation. Small-scale gold mining in the Amazon: the cases of Bolivia, Brazil, Colombia, Peru and Suriname. Cuadernos del CEDLA No. 26. Amsterdam. 2013.

DRAKE, P. L.; ROJAS, M.; REH, C. M.; MUELLER, C. A.; JENKINS, F. M. Occupational exposure to airborne mercury during gold mining operations near El Callao, Venezuela. Int Arch Occup Environ Health. v. 74, n. 3, p. 206-212, 2001. Disponível em: < https://www.ncbi.nlm.nih.gov/pubmed/11355295>.

DRISCOLL, C. T.; MASON, R. P.; CHAN, H, M.; JACOB, D. J.; PIRRONE, N. Mercury as a Global Pollutant: Sources, Pathways, and Effects. Environmental Science \& Technology, v. 47, n.10, p.4967- 4983, 21 Maio 2013. American Chemical Society (ACS). http://dx.doi.org/10.1021/es305071v.

DUTRA, M. D. S.; JESUS, I. M.; SANTOS, E. C. O.; LIMA, M. D. O.; MEDEIROS, R. L. F.; CAVADAS, M.; LUIZ, R. R.; CÂMARA, V. M. Longitudinal assessment of mercury exposure in schoolchildren in an urban area of the Brazilian Amazon. Cadernos de Saúde Pública, [s.l.], v. 28, n. 8, p.1539-1545, ago. 2012. FapUNIFESP (SciELO). http://dx.doi.org/10.1590/s0102-311x2012000800012.

DUTRA, M. D. S.; CAVADAS, M.; JESUS, I. M.; SANTOS, E. O.; SILVA, E. A.; CÂMARA, V. M. Hearing thresholds in children exposed to mercury in the prenatal period. Jornal da Sociedade Brasileira de Fonoaudiologia. [online]. 2012, vol.24, n.4, pp.322-326. ISSN 2179-6491. Disponível em:<http://dx.doi.org/10.1590/S2179$64912012000400006>$.

FARIAS, L. A.; SANTOS, N. R. S.; FAVARO, D. I. T.; BRAGA, E. S. Total hair mercury in children from a coastal population in Cananéia, São Paulo State, Brazil. Cadernos de Saúde Pública [online]. v.24, n.10, p.2249-2256 2008. ISSN 1678-4464. Disponível em:<http://dx.doi.org/10.1590/S010211X2008001000006 >.

FUJIMURA, M.; MATSUYAMA, A.; HARVARD, J.P.; BOURDINEAUD, J.P.; NAKAMURA, K. Mercury contamination in Humans in Upper Maroni, French Guiana 
Between 2004 and 2009. Bull Environ Contam Toxicol (2012) 88:135-139, LLC 2011. Disponível em:< DOI 10.1007/s00128-011-0497-3>.

FRÉRY, N.; MAURY-BRACHET, R.; MAILLOT, E.; DEHEEGER, M.; MÉRONA, B.; BOUDOU, A. Gold-mining activities and mercury contamination of native Amerindian communities in French Guiana: Key role of fish in dietary uptake. Environmental Health Perspectives, v. 109, n. 5, p. 449-456, 2001.

GAIOLI, M.; AMOEDO, D.; GONZÁLEZ, D. Impacto del mercurio sobre la salud humana y el ambiente. Arch Argent Pediatr, v. 110, n. 3, p. 259-264, 2012. Disponível em: < http://dx.doi.org/10.5546/aap.2012.259>.

GERHARDT, T. E.; SILVEIRA, D. T. (Orgs). Métodos de pesquisa. Porto Alegre: Editora da UFRGS, 120 p., 2009.

GONZÁLEZ-CARRASCO, V.; VELASQUEZ-LOPEZ, P. C.; OLIVERO-VERBEL, J.; PÁJARO-CASTRO, N. Air mercury contamination in the gold mining town of Portovelo, Ecuador. Bulletin of Environmental Contamination and Toxicology, v. 87, n. 3, p. 250-253, 2011. Disponivel: < DOI: 10.1007/s00128-011-0345-5>.

GRIGOLETTO, J. C.; OlIVEIRA, A. D. S.; MUÑOZ, S. I. S.; ALBERGUINI, L. B. A.; TAKAYANAGUI, A. M. M. Exposição ocupacional por uso de mercúrio em odontologia: uma revisão bibliográfica. Ciência \& Saúde Coletiva, v. 13, n. 2, p. 533-542, 2008.

GUZZI, G.; PIGATTO, P. D. Occupational exposure to mercury from amalgams during pregnancy. Occupational and environmental medicine, v. 64 , n. 10, p. 715-716; discussion 715-716, 2007.

HAGAN, N.; ROBINS, N.; HSU-KIM, H.; HALABI, S.; GONZALES, R. D. E.; RICHTER, D.; VANDENBERG, J. (2013). Residential mercury contamination in adobe brick homes in Huancavelica. Peru. PLoS One, 8, e75719. Disponível em: $<10.1371$ /journal.pone.0075179>.

HARARI, R.; HARARI, F.; GERHARDSSON, L.; LUNDH, T.; SKERFVING, S.; STRÖMBERG, U.; BROBERG, K. Exposure and toxic effects of elemental mercury in gold-mining activities in Ecuador. Toxicology Letters, v. 213, n. 1, p. 75-82, 2012. Elsevier Ireland Ltd. Disponível em: <http://dx.doi.org/10.1016/j.toxlet.2011.09.006>.

HINTON, J. J.; VEIGA, M. M.; BEINHOFF, C.; Women and Artisanal Mining: Gender Roles and the Road Ahead. Chapter 11 - book: The Socio-Economic Impacts of Artisanal and Small-Scale Mining in Developing Countries Ed. G. Hilson, Pub. A.A. Balkema, Swets Publishers, Netherlands, 2003. p. 739. Disponível em:< http://siteresources.worldbank.org/INTOGMC/Resources/3360991163605893612/hinton rolereview.pdf $>$.

HURTADO, J.; GONZALES, G. F.;STEENLAND, K. Mercury Exposures in Informal Gold Miners and Relatives in Southern Peru. International Journal of Occupational and ENCICLOPÉDIA BIOSFERA, Centro Científico Conhecer - Goiânia, v.13 n.24; p. 1362 2016 
Environmental Health, 12:4, 340-345 (2013). Disponível em:< http://dx.doi.org/10.1179/oeh.2006.12.4.340 >.

LEMIRE, M.; MERGLER, D.; FILLION, M.; PASSOS, C. J. S.; GUIMARÃES, J. R. D.; DAVIDSON, R.; LUCOTTE, M. Elevated blood selenium levels in the Brazilian Amazon. Science of the Total Environment, v. 366, n. 1, p. 101-111, 2006. Disponível em: < DOI: 10.1016/j.scitotenv.2005.08.057>.

LEMOS, A.; MALASPINA, F.G.; SALLAS, J.; URIBE, M. V.; GUTIÉRREZ, S. A. Cooperação técnica entre Brasil, Bolívia e Colômbia para o fortalecimento da vigilância em saúde de populações expostas ao mercúrio. Cap 8: Sistemas de informação para notificação de intoxicação e exposição humana a substâncias químicas. Brasília: Organização Pan-Americana da Saúde, 2011.

MARRERO, P. S.; RICHANI, J.; ROJAS, G.; QUERALES, M.; GONZÁLEZ, S. Exposición ambiental al mercurio y valores en orina de los habitantes de la comunidad Boca de Yaracuy , ubicada en la costa centro-norte de Venezuela. Gac Méd Caracas, v. 119, n. 4, p. 315-320, 2011.

MARQUES, R.C.; DÓREA, J. G.; BASTOS, W.R.; REBELO, M.F.; FONSECA, M.F.; MALM. O. Maternal mercury exposure and neuro-motor development in breastfed infants from Porto Velho (Amazon), Brazil. Science of The Total Environment, v. 210, p. 51-60, 2007. Disponível em: < http://dx.doi.org/10.1016/j.ijheh.2006.08.001>.

MONRROY, S.J.; RODRIGUEZ, R.W.; ROULET, M.; BENEFICE, E. Lifestyle and mercury contamination of Amerindian populations along the Beni river (lowland Bolivia). Journal of Environmental Health, n. 71 (4), p. 44-50, 2008.Disponível em:< http://hal.ird.fr/ird-00339132 >.

PASSOS C. J. S.; MERGLER, D. Human mercury exposure and adverse health effects in the Amazon: a review. Caderno de Saúde Pública [online]. 2008, v.24, suppl.4, p 503-520. ISSN 1678-4464. Disponível em:< http://dx.doi.org/10.1590/S0102$311 \times 2008001600004>$.

PASSOS, C. J .S.; MERGLER, D.; LEMIRE, M.; FILLION, M.; RÉMY, J.; GUIMARÃES, $D$. Lifestyle and mercury contamination of Amerindian populations along the Beni river (lowland Bolivia). Journal of Environmental Health, v. 373, ISSUE 1, p. 68-76, 2007. Disponível em:< http://dx.doi.org/10.1016/j.scitotenv.2006.11.015 >.

PENNA FILHO, P. Reflexões sobre o Brasil e os desafios Pan-Amazônicos. Revista Brasileira de Política Internacional [s.i.], v. 56, n. 2, p.94-111, dez. 2013. FapUNIFESP (SciELO). Disponível em:<http://dx.doi.org/10.1590/s003473292013000200006>.

PEPLOW, D.; AUGUSTINE, S. Community-directed risk assessment of mercury exposure from gold mining in Suriname. Revista Panamericana de Salud 
Publica [online]. 2007, v.22, n.3, pp.202-210. ISSN 1680-5348. Disponível em:< http://dx.doi.org/10.1590/S1020-49892007000800007>.

PEPLOW, D; AUGUSTINE, S. Community led assessment of risk from exposure to mercury by native Amerindian Wayana in southeast Suriname. Journal of Environmental and Public Health, 2012. Disponível em:<http://dx.doi.org/10.1155/2012/674596>.

PINHEIRO, M. C. N.; NAKANISHI, J.; OIKAWA, T.; GUIMARÃES, G.; QUARESMA, M.; CARDOSO, B.; AMORAS, W.W.; HARADA, MASAZUME.; MAGNO, C.; VIEIRA, J. L F.; XAVIER, M. B.; BACELAR, D. R. Methylmercury human exposure in riverine villages of Tapajos basin, Pará State, Brazil. Revista da Sociedade Brasileira de Medicina Tropical [online]. 2000, v.33, n.3, p.265-269. ISSN 0037-8682. Disponível em: < http://dx.doi.org/10.1590/S0037-86822000000300005>.

RAVENA, N.; CAÑETE, V. R. Reflexões sobre a integração Pan-Amazônica: o papel da Organização do Tratado de Cooperação Amazônica (OTCA) na regulação da água. Revista Brasileira de Estudos Urbanos e Regionais, Belém, v. 9, n. 7, p.131144, maio 2007. Disponível em: <http://unuhospedagem.com.br/revista/rbeur/index.php/rbeur/article/viewFile/175/159>.

ROCHA NETO, A. D. Convenção de Minamata: Seminário de atualização sobre o mercúrio. 2013.2 Disponível em: <http://www.fundacentro.gov.br/Arquivos/sis/EventoPortal/AnexoPalestraEvento/Semina rioMercurio_Fundacentro_ConvencaoMinamata_6e7 08 13.pdf>.

ROJAS, J.; SEIJAS, D.; AGREDA, O.; RODRÍGUEZ, M. Biological monitoring of mercury exposure in individuals referred to a toxicological center in Venezuela. Science of The Total Environment, 2006, v.354, n.4, p. 278-285. ISSN 2-3. Disponível em: < http://dx.doi.org/10.1179/oeh.2006.12.4.340>.

ROJAS, M. Exposición ambiental y humana al mercurio en Venezuela: 2004-2008. Red de Revistas Científicas de América Latina y El Caribe, España y Portugal: Salus, Venezuela, v. 14, n. 2, p.33-40, ago. 2010. Disponível em: <http://www.redalyc.org/articulo.oa?id=375939014009>.

SANTANA, V.; MEDINA, G.; TORRE, A. El Convenio de minamata sobre el mercurio y su implementación en la región de América Latina y el Caribe. 2014.

SANTOS, E. C.; CÂMARA, V. M.; BRABO, E. S.; LOUREIRO, E. C.B.; JESUS, I. M.; F.; FAYAL, K.; SAGICA, F. Mercury exposure among Pakaanóva Indians, Amazon Region, Brazil. Cad. Saúde Pública [online]. 2003, v..19, n.1, p.199-206. ISSN 16784464. Disponível em:< http://dx.doi.org/10.1590/S0102-311X2003000100022>.

SILVA, A. S. D. Organização pan-americana da saúde. 2011. 
STETE, J.; GASTAÑAGA, M. C.; FIESTAS, V.; OBLITAS, T.; SABASTIZAGAL, I.; LUCERO, M.; ABADIE, J. M.; MUNÕZ, M.E.; VALVERDE, A.; SUAREZ, M. Enfermedades transmisibles, salud mental y exposición a contaminantes ambientales en población aledaña al proyecto minero Las Bambas antes de la fase de explotación, Perú 2006. Rev. perú. med. exp. salud publica [online]. 2010, v.27, n.4, pp. 512-519. ISSN 1726-4634.

TELMER, K.H.; VEIGA, M.M. World emissions of mercury from artisanal and small scale gold mining. in: mercury fate and transport in the global atmosphere: emissions, measurements and models (Pirrone N, Mason R, eds.). New York, NY: Springer (2009).

TINÔCO, A. A .P.; AZEVEDO, I. C. D. D.; MARQUES, E. A. G.; MOUNTEER, A. H.; MARTINS, C. D. P.; NASCENTES. R.; REIS, E. R.; NATALINO, R. Avaliação de contaminação por mercúrio em Descoberto, MG, Engenharia Sanitária e Ambiental, 15 (4), 305-314, 2010.

TRASANDE, L.; CORTES, J. E.; LANDRIGAN, P. J.; ABERCROMBIE, M. I.; BOPP, R. F.; CIFUENTES, E. Methylmercury exposure in a subsistence fishing community in Lake Chapala, Mexico: an ecological approach. Environmental Health, 9 (1), 2010. Disponível em: <DOI: 10.1186/1476-069X-9-1>.

WASSERMAN, E. Environment, health, and gender in Latin America: trends and research issues. Environmental research, v. 80, n. 3, p. 253-273, 1999. Disponível em: < DOI:10.1006/enrs.1998.3943>.

YARD, E. E.; HORTON, J.; SCHIER, J. G.; CALDEWELL, K.; SANCHEZ, C.; LEWIS, L.; GASTANAGA, C. Mercury Exposure Among Artisanal Gold Miners in Madre de Dios, Peru: A Cross-sectional Study. Journal of Medical Toxicology, v. 8, n. 4, p. 441-448, 2012. Disponível em:<DOI: 10.1007/s13181-012-0252-0>.

VISNJEVEC, A. M.; KOCMAN, D.; HORVAT, M. Human mercury exposure and effects in Europe.Environmental Toxicology And Chemistry, [s.I.], v. 33, n. 6, p.1259-1270, abr/2014. Wiley-Blackwell. Disponível em: <http://dx.doi.org/10.1002/etc.2482>. 